\title{
Single-Pass Albumin Dialysis in the Treatment of Children with Liver Failure
}

\author{
Johannes Holle ${ }^{a, b}$ Alexander Gratopp ${ }^{a}$ Sophie Balmer ${ }^{a}$ Verena Varnholt ${ }^{a}$ \\ Stephan Henning ${ }^{b}$ Philip Bufler $^{b}$ Dominik Müller ${ }^{b}$ Leonard Rosenfeld ${ }^{a}$ \\ ${ }^{a}$ Department of Pediatric Pulmonology, Immunology and Intensive Care Medicine, Charité-Universitätsmedizin \\ Berlin, Berlin, Germany; bepartment of Pediatric Gastroenterology, Nephrology and Metabolic Diseases, \\ Charité-Universitätsmedizin Berlin, Berlin, Germany
}

\section{Keywords}

Liver failure - Liver transplantation - Liver support therapy · Chronic liver failure $\cdot$ Hepatic encephalopathy · Albumin dialysis

\begin{abstract}
Background and Aims: Acute and acute on chronic liver failure are life-threatening conditions, and bridging to transplantation is complicated by a paucity of suitable organs for children. While different modalities of extracorporeal liver support exist, their use in children is complicated by a large extracorporeal volume, and data on their use in children is limited. The aim of this analysis was to investigate the efficacy and safety of single-pass albumin dialysis (SPAD) in children with liver failure. Methods: Retrospective medical chart review of pediatric patients with liver failure treated with SPAD. The decrease in hepatic encephalopathy (HE) and the serum levels of bilirubin and ammonia were measured to determine efficacy. Adverse events were documented to assess safety. Results: Nineteen pediatric patients with a median age of 25.5 months and a median body weight of $11.9 \mathrm{~kg}$ were treated with SPAD between January 2011 and March 2018. Total bilirubin $(p<0.001)$ and ammonia $(p=0.02)$ significantly decreased after treatment with SPAD. As clinical outcome parameter, HE significantly improved ( $p=0.001)$.
\end{abstract}

Twelve patients were bridged successfully to liver transplantation. In all patients, 71 SPAD sessions were run. Clotting in the dialysis circuit was observed in $49 \%$ of all sessions. Heparin and citrate were used for anticoagulation and were significantly superior to dialysis without any anticoagulation ( $p=0.03)$. Transfusion of packed blood cells (57\%) and catecholamine therapy (49\%) were frequently necessary. Conclusions: Treatment with SPAD was effective in detoxification, as measured by significant improvement of $\mathrm{HE}$ and clearance from surrogate laboratory parameters.

(c) 2019 S. Karger AG, Basel

\section{Introduction}

Acute and chronic liver failures are life-threatening conditions. Liver transplantation (LTX) has become the standard therapy for end-stage liver failure in children with 5-year survival rates of $85 \%$ [1]. However, acute liver failure (ALF) may be reversible and the availability of suitable organs is limited. Therefore, strategies of extracorporeal liver support have been developed to bridge to recovery or transplantation. The rationale of such extracorporeal liver support systems is to reduce endogenous water-soluble (e.g., ammonia) as well as protein-bound toxins (e.g., bilirubin and aromatic amino acids), and

\section{KARGER}

(c) 2019 S. Karger AG, Basel

E-Mail karger@karger.com

www.karger.com/bpu
Dr. med. Johannes B. Holle

Department of Pediatric Gastroenterology, Nephrology and Metabolic Diseases Charité-Universitätsmedizin Berlin

Augustenburger Platz 1, DE-13353 Berlin (Germany)

E-Mail johannes-benjamin.holle@ charite.de 
thereby attenuate endotoxinemia and hepatic encephalopathy (HE) [2-5].

Water-soluble substances can be effectively removed bycontinuous venovenous hemodiafiltration (CVVHDF) [6]. In contrast, protein-bound substances cannot be effectively eliminated by conventional dialysis techniques such as CVVDHF. They need a comparable acceptor on the opposite side of the membrane, that is, in the dialysate to be eliminated from the blood. To overcome this problem, albumin is added as a scavenger in the dialysate bath.

Single-pass albumin dialysis (SPAD) uses a dialysis machine capable of CVVHDF. Blood flows through a high-flux dialysis filter, and the dialysate contains a variable concentration of human albumin [7].

Our aim was to evaluate the performance of SPAD in clinical practice in a representative cohort of pediatric patients with liver failure. We hypothesize that SPAD effectively removes water-soluble and protein-bound toxins represented by ammonia and bilirubin, respectively, as surrogate parameters and improves $\mathrm{HE}$ as clinical endpoint. As SPAD is used in critically ill children with possible bleeding disorders and hemodynamic instability, we were especially interested in analyzing adverse events with a particular focus on the choice of anticoagulant applied.

\section{Patients and Methods}

All patients with ALF or acute on chronic liver failure who were treated with SPAD on our pediatric intensive care unit from January 2011 until March 2018 were included in the study. The study was performed in accordance with federal and state laws and recommendations of the local data safety office. The Institutional Review Board of Charité-Universitätsmedizin Berlin waived the need for approval due to the retrospective nature of the study.

Clinical and laboratory data were extracted from patients' records. These included age, gender, body weight, and diagnosis as well as grade of HE according to the West Haven classification [8]. Laboratory data included bilirubin, ammonia, creatinine, and international normalized ratio. Furthermore, the "model of endstage liver disease" score and "pediatric end-stage liver disease" score for children below the age of 12 years were calculated $[9,10]$.

Laboratory data were collected at least once per day. Clinical data, especially grade of HE, were assessed once per shift, usually 3 times per day.

\section{SPAD Setting}

Indication for the initiation of SPAD was at least one of the following criteria: $\mathrm{HE}$ (grade $\geq 2$ ), elevated bilirubin $(>400 \mu \mathrm{mol} / \mathrm{L})$, hepatorenal syndrome according to the definition of Bozanich and Kwo [11] or elevated ammonia ( $>200 \mu \mathrm{mol} / \mathrm{L})$. A central venous double-lumen catheter was placed in the jugular vein, subclavian vein or femoral vein. The minimum catheter size was 6.5 French.
SPAD was performed as a post-dilution CVVHDF with Plasauto Sigma (Asahi Kasei Kuraray Medical Co., Ltd., Chiyoda Ku, Japan) using an Ultraflux EMiC2 dialyzer (Ultraflux ${ }^{\circledR}$ dialyzer, steam-sterilized, covering $1.8 \mathrm{~m}^{2}$ surface area, Fresenius Polysulfone ${ }^{\circledR}$ membrane, Fresenius Medical Care GmbH, Bad Homburg, Germany). Dialyzer $(130 \mathrm{~mL})$ plus dialysis circuit $(69 \mathrm{~mL}$ for children and adolescents and $47 \mathrm{~mL}$ for children with a body weight below $20 \mathrm{~kg}$ ) were partially filled with packed red blood cells in case of hemodynamic instability. Blood flow rates between 3 and $5 \mathrm{~mL} /$ $\mathrm{kg} *$ min were achieved. As dialysis solution, either CiCa Dialysate K2 (Fresenius Medical Care GmbH, Bad Homburg, Germany) for citrate anticoagulation or Accusol 35 (Baxter Healthcare Ltd., Thetford, UK) for heparin anticoagulation were used. A 3-5\% albumin solution was produced online using one additional roller pump for human albumin 20\% (Baxter Healthcare Ltd., Thetford, UK) which was then mixed via Y connector with the CiCa Dialysate K2 or Accusol 35 dialysis solution, as described previously by our group [7]. This albumin solution for hemodialysis was set continuously at flow rates of $20-40 \mathrm{~mL} / \mathrm{kg} * \mathrm{~h}$. The continuous venovenous hemofiltration was performed with Accusol 35 or CiCa K2 dialysis solution as substitution solution.

Anticoagulation was administered either by continuous heparin or regional citrate anticoagulation (RCA). The choice was made individually by the treating physician before starting SPAD treatment. There was no treatment algorithm for the choice and dosage of the anticoagulant. Patients that were considered subjectively to have a high risk of bleeding were started with RCA or without any anticoagulation. Patients with citrate accumulation, indicated by an increased total/ionized calcium ratio $>2.5$ or uncontrollable alkalosis or acidosis, were switched to heparin anticoagulation.

\section{Outcome Analysis}

Effectiveness of SPAD was investigated by comparison of clinical (HE) and laboratory data (ammonia, bilirubin) within $24 \mathrm{~h}$ prior and after initiation of SPAD. The final outcome (transplantation, survival) was registered.

The duration of dialysis sessions under different modes of anticoagulation, reasons for interruptions as well as complications were recorded. These included blood transfusions, need for vasoactive medication, allergic reactions, and bleeding complications.

Bleeding was classified as "life-threatening" following criteria proposed for children with hemorrhagic complications in dialysisdependent acute kidney failure [12]. In our setting, a hemorrhagic complication was defined as "life-threatening" (1) when blood hemoglobin fell by $>2 \mathrm{~g} / \mathrm{dL} / 6 \mathrm{~h}$ or required transfusion of $>20 \mathrm{~mL}$ packed red cells per kilogram body weight in $24 \mathrm{~h}$, (2) when bleeding involved vulnerable locations, for example, brain, pericardium, lung or (3) when bleeding significantly affected vital parameters requiring an altered use of catecholamines. Non-life-threatening episodes included bleeding from catheter insertion site, mucosal bleeding (nose, mouth) without need for intervention, tracheal bleeding without respiratory decompensation, gastrointestinal bleeding without need for intervention, skin bleeding, and hematuria without vesical tamponade.

\section{Statistical Analysis}

Microsoft Excel (2013) and GraphPad Prism (7.04; GraphPad Software, La Jolla, CA, USA) were used for data collection and statistical analysis. Normality of variables was tested by Shapiro-Wilk 
Table 1. Demographic characteristics and clinical outcome of patients treated with SPAD

\begin{tabular}{|c|c|c|c|c|c|}
\hline Patient & Age, months & Weight, kg & Gender & Outcome & Cause of death \\
\hline $1 \mathrm{a}$ & 25 & 11 & Male & Recovery & \\
\hline $1 b$ & 26 & 12 & Male & \# & Sepsis \\
\hline 2 & 205 & 75 & Male & LTX & \\
\hline 3 & 205 & 62 & Female & \# & ARDS \\
\hline 4 & 1 & 4.2 & Male & Recovery & \\
\hline 5 & 1 & 5.4 & Male & LTX, \# & MOF \\
\hline 6 & 24 & 9.0 & Male & Re-LTX & \\
\hline 7 & 205 & 75 & Female & LTX & \\
\hline 8 & 199 & 52 & Male & LTX & \\
\hline 9 & 2 & 3.5 & Female & LTX & \\
\hline 10 & 162 & 80 & Female & LTX & \\
\hline 11 & 22 & 12 & Male & LTX & \\
\hline 12 & 39 & 15 & Male & LTX & \\
\hline 13 & 0.5 & 2.6 & Male & $\#$ & MOF \\
\hline 14 & 6 & 6 & Male & \# & $\mathrm{CE}$ \\
\hline $15 \mathrm{a}$ & 58 & 27 & Female & Recovery & \\
\hline $15 b$ & 58 & 27 & Female & \# & Sepsis \\
\hline 16 & 24 & 10 & Female & LTX & \\
\hline 17 & 4 & 6.5 & Male & \# & MOF \\
\hline $18 \mathrm{a}$ & 155 & 44 & Female & LTX & \\
\hline $18 \mathrm{~b}$ & 155 & 44 & Female & Recovery & \\
\hline 19 & 4 & 7.6 & Female & LTX, \# & MOF \\
\hline
\end{tabular}

SPAD, single-pass albumin dialysis; \#, death; LTX, liver transplantation; ARDS, acute respiratory distress syndrome; MOF, multiple organ failure; CE, cerebral edema.

normality test. Paired $t$ test and Wilcoxon matched-pairs signed rank test were used to compare variables. The association of body weight with the duration of dialysis sessions was analyzed using Pearson correlation and visualized using a regression line in a scatter plot. $p$ values $<0.05$ were considered as statistically significant. Data are shown as mean \pm SD or median (interquartile range [IQR]).

\section{Results}

Nineteen pediatric patients ( 7 girls, 12 boys) with ALF or acute on chronic liver failure were treated with SPAD. Patient demographic characteristics are shown in Table 1 and clinical and laboratory characteristics before initiation of SPAD are shown in online supplementary Table 1 (for all online suppl. material, see www.karger.com/ doi/10.1159/000502938; supplemental data available upon request at the corresponding author). The median age of the patients was 25.5 (152.8) months and the median body weight was 11.85 (39.7) $\mathrm{kg}$. The most common diagnosis leading to the prescription of SPAD was ALF of unknown origin in 7 cases, followed by gestational alloimmune liver disease in 3 cases.

SPAD in Children with Liver Failure
In 3 patients, SPAD treatment was discontinued for $>48 \mathrm{~h}$ due to transient recovery of liver function (patients 1 and 15) or transplantation (patient 18) and restarted when liver function deteriorated again.

\section{SPAD Settings}

Data of dialysis modalities are shown in Table 2. The median duration of SPAD was $65.5 \mathrm{~h}$ (IQR 76.5; minimum 5; maximum 369). In total, 71 SPAD sessions were performed. The median session time was 21.5 (IQR 30.5) h. The median blood flow was 3.8 (IQR 1.7 ) $\mathrm{mL} / \mathrm{kg} * \mathrm{~min}$, median substitution fluid flow was 31.5 (IQR 21.25 ) mL/ $\mathrm{kg} * \mathrm{~h}$, and median dialysate flow was 26 (IQR 17.25) mL/ $\mathrm{kg} * \mathrm{~h}$ with a median of $4 \%$ concentration of albumin.

Only $17 / 71$ sessions (23.4\%) were longer than $36 \mathrm{~h}$, while max. 48 hours were aspired for scheduled filter and system replacement. The patients' weights had no influence on session time $\left(r^{2}=0.02 ; p=0.28\right.$; Fig. 1a).

There was a significant correlation between anticoagulation and the duration of dialysis sessions (Fig. 1b). Cycles in which SPAD was conducted without anticoagulation $(n=7)$ had significant shorter cycle duration than patients with heparin $(n=41 ; p=0.03)$ or RCA $(n=23$; 


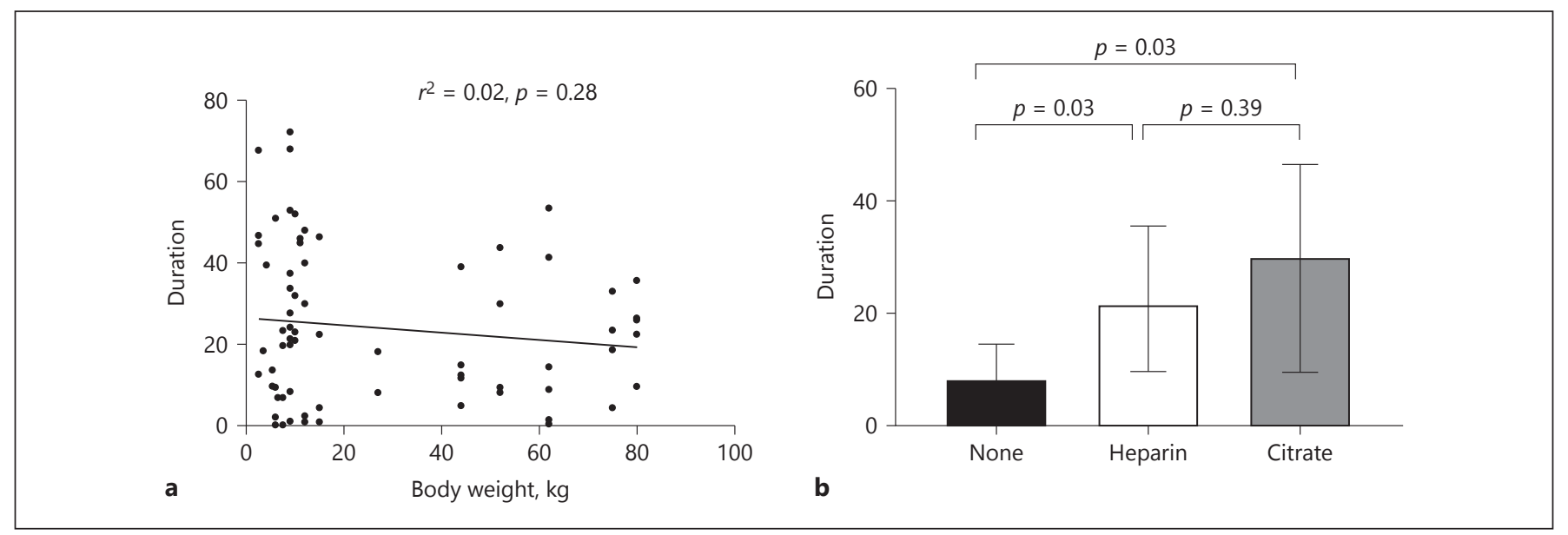

Fig. 1. Influence of body weight and anticoagulation on SPAD duration. a Association of duration of each single session and patient's body weight. There was no significant association between both parameters analyzed by Pearson correlation and visualized by linear regression. $\mathbf{b}$ Influence of anticoagulation on the duration of SPAD sessions. Patients without any anticoagulation had significant lower session times than patients with heparin or citrate anticoagulation. Bars indicate median values, whiskers indicate IQR.

Table 2. Dialysis data of all patients treated with SPAD

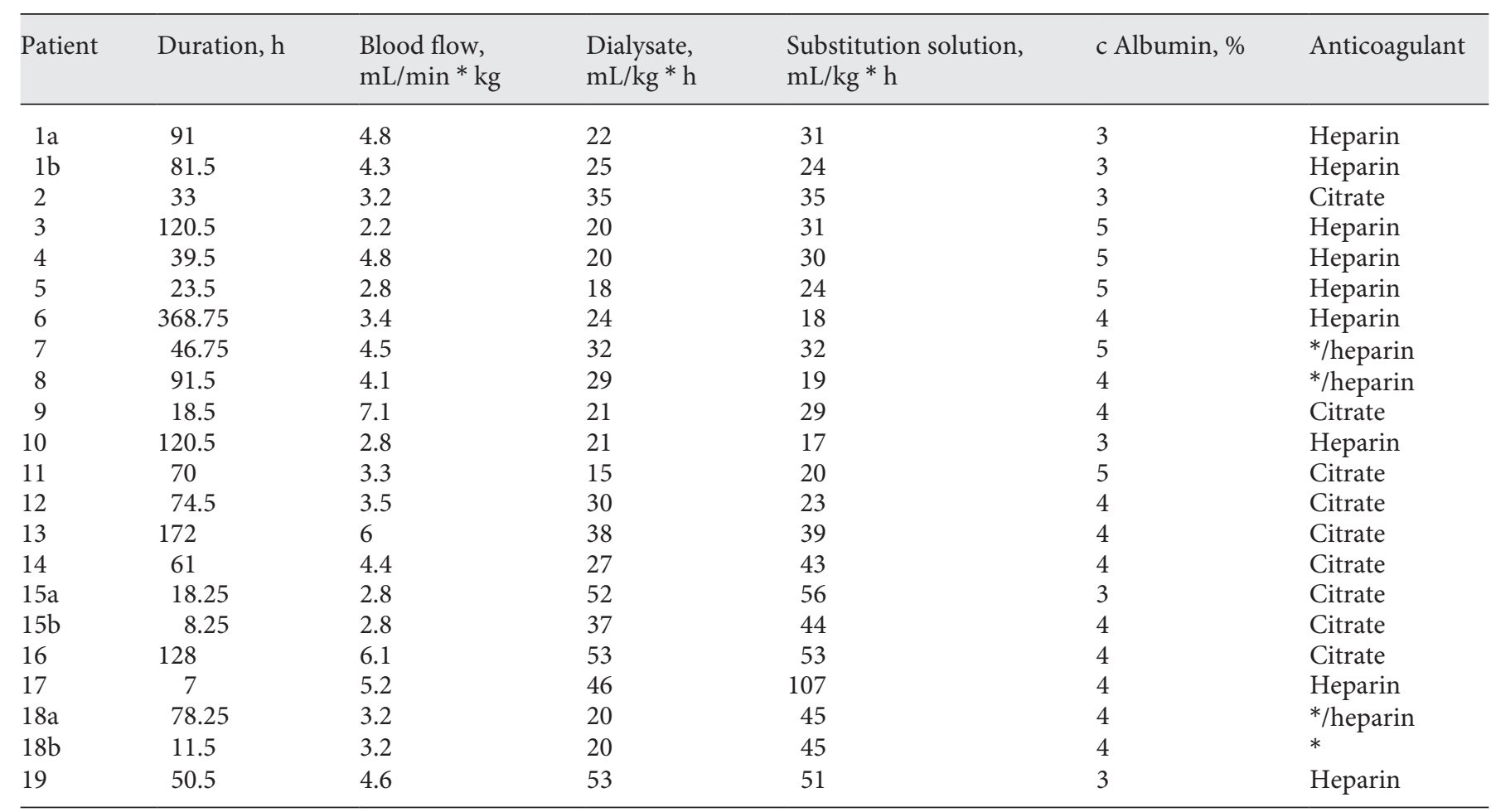

* No anticoagulation.

SPAD, single-pass albumin dialysis; c Albumin, concentration of albumin in the dialysate. 


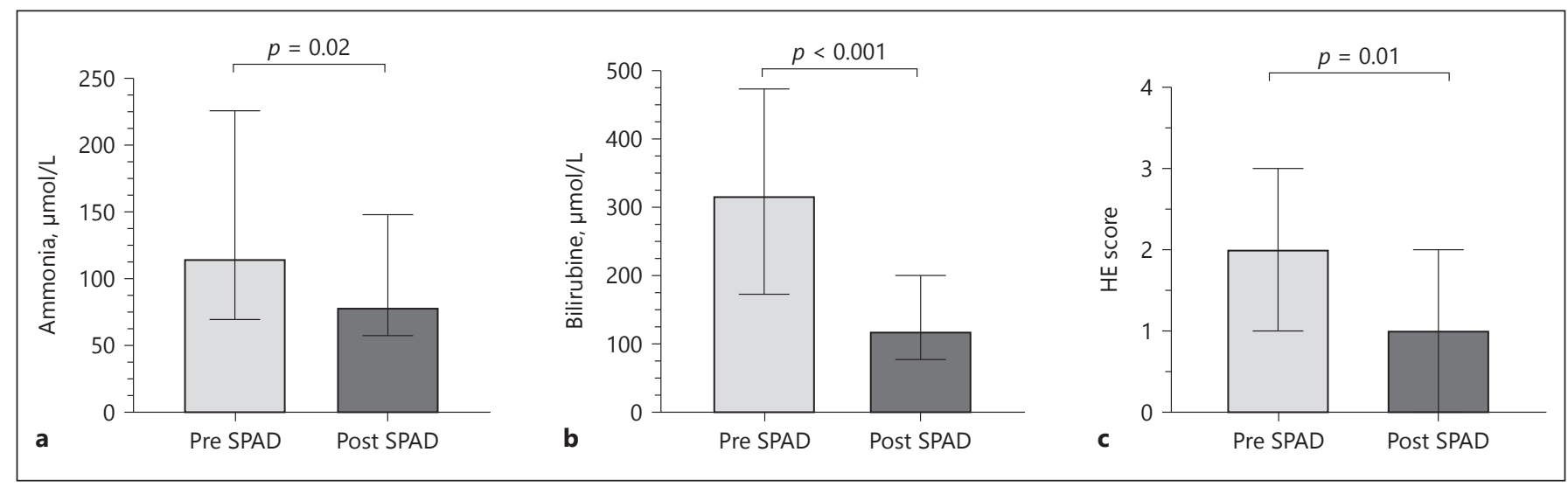

Fig. 2. Efficacy of SPAD. Efficacy of SPAD according to elimination of ammonium (a), bilirubin (b), and improvement of HE (c). Bars indicate median and interquartile range. $p$ value indicates significance of difference before and after SPAD. SPAD, single-pass albumin dialysis; HE, hepatic encephalopathy.

$p=0.03)$. The most common cause for the termination was clotting in the dialysis circuit (49\%). About $14 \%$ of sessions were discontinued for scheduled filter or dialysis machine change and $13 \%$ due to LTX.

\section{Efficacy of SPAD}

We observed a significant decrease of surrogate laboratory markers and clinical grade of encephalopathy (Fig. 2). Ammonia levels decreased significantly during therapy (median decrease $36.5 \mu \mathrm{mol} / \mathrm{L}, p=0.02$ ). Bilirubin decreased significantly in all patients (median decrease $193 \mu \mathrm{mol} / \mathrm{L} ; p<0.001$ ). There were no significant differences in the bilirubin clearance according to the albumin concentration in the dialysate bath, which ranged between 3 and 5\% (online suppl. Fig. 1, Supplemental data available upon request to the corresponding author). HE improved or remained stable in all patients during SPAD (median improvement 1.0, mean improvement $0.84 ; p=0.01)$.

\section{Outcome}

Clinical outcome is shown in Table 1. In our, cohort 8 of 19 patients deceased. Two patients deceased after LTX, 6 patients while waiting for a graft, having a contraindication for transplantation or being switched to palliative care. Twelve of 19 patients (63\%) were bridged to LTX. After LTX, 1 patient underwent another session of SPAD due to insufficient graft function; after $12 \mathrm{~h}$, SPAD was discontinued. Two patients died 3 weeks and 3 months after LTX, respectively. All other patients had sufficient liver function on discharge from our pediatric intensive care unit. Three patients displayed temporary recovery of liver function during SPAD. The first (patient 1a) had neuroblastoma-amplified sequence mutation (OMIM 608025) with acute onset of liver failure during a febrile infection and recovered after $46 \mathrm{~h}$ of SPAD. He deceased during a second episode of liver failure. A second child (patient 4) with gestational alloimmune liver disease recovered after $40 \mathrm{~h}$ of SPAD; the third child (patient 15a) recovered temporarily and albumin dialysis was discontinued before liver function deteriorated again. Eventually, the child deceased as transplantation was not possible due to metastatic malignant disease.

\section{Complications}

There were no allergic reactions during SPAD. Most common complications were non-life-threatening bleedings. During SPAD, bleedings in the ear/nose/throat area $(n=20)$, catheter insertion site $(n=18)$, gastrointestinal bleeding $(n=9)$, hematuria $(n=5)$, and skin bleedings $(n=6)$ were reported. One patient suffered from a lifethreatening pulmonary hemorrhage with secondary cardiorespiratory decompensation leading to the discontinuation of SPAD. Inappropriately rapid re-transfusion of blood in a small child $(4.2 \mathrm{~kg})$ at the end of dialysis was identified as the reason for this complication.

Two out of 10 patients, in who RCA was initiated, were switched to heparin anticoagulation due to citrate accumulation. There were no differences in the frequency of minor hemorrhages between the heparin and the RCA groups.

No patient died due to a direct complication of SPAD. In 53/71 sessions (75\%) packed blood cells were substituted and in 35/71 sessions (49\%) catecholamine therapy was necessary to support sufficient systemic circulation. 


\section{Discussion}

In this analysis, we assessed the performance of SPAD under clinical conditions in pediatric intensive care treatment of liver failure. SPAD is administered to patients with liver failure with the intention to bridge to transplantation or hepatic recovery, although we know little about its benefits. To date, this is the largest series of children treated with SPAD for liver failure. With 13/19 patients $(68 \%)$ with a body weight of $15 \mathrm{~kg}$ or less, our data reflect the unique features of a pediatric cohort.

We are well aware of the fact that our retrospective evaluation without control group cannot answer the most meaningful clinical outcomes such as a possible benefit in survival, mechanical ventilation or vasopressor support in comparison to a cohort undergoing standard medical treatment without SPAD. However, we demonstrate that SPAD significantly eliminates water-soluble compounds, such as ammonia, and protein-bound substances, such as bilirubin. However, the most important clinical benefit is the significant improvement of HE.

The comparison of our results to available data is complicated by the fact that various modalities of extracorporeal liver support exist and most data are derived from adult patients. Hence, a direct comparison is impossible. A commercially available and extensively studied system using albumin dialysis is the molecular adsorbents recirculating system (MARS Baxter, Stockholm, Sweden, 1996) [13]. To minimize extracorporeal circulation, a volume-adapted pediatric MARS system (MARSmini) has been developed for use in children [14, 15]. Different approaches use a combination of plasmapheresis and hemodialysis, performed simultaneously with a plasma separator and a high-flux filter configured serially in children with ALF $[15,16]$.

In children, most reports on extracorporeal liver support systems in children focus on MARS [15, 17-20]. Only few reports exist about the use of SPAD as a detoxification device, especially in Wilson's disease and drug intoxication $[7,21,22]$.

Regarding the clinical efficacy of SPAD, we could corroborate the reduction of HE reported by our study group in a previous case series [7]. This effect is comparable to MARS whereas there is no comparable data on combined plasma exchange and hemodialysis $[7,16,23,24]$.

Respecting the improvement of laboratory surrogate parameters, the effective elimination of bilirubin with SPAD in our cohort seems to be comparable with results from children treated with MARS and with combined plasma exchange and hemodialysis $[15,16,19]$.
In contrast to our data, a meta-analysis on albumin dialysis failed to demonstrate a significant decrease in ammonia [25]. As ammonia is similar to urea in terms of diffusive clearance [6], this could be explained by an insufficient dose of hemodialysis.

Currently, there is only one open randomized trial demonstrating a potential survival benefit of high-volume plasma exchange in adult patients with acute liver disease [26]. For MARS, despite the positive effects on short-term toxin removal and HE, a survival benefit could not be demonstrated in adult patients $[25,27]$. There is no comparable pediatric data.

Despite the lack of head-to-head comparisons between SPAD and other liver replacement methods in children, we postulate SPAD as a possibly effective treatment in children with liver failure. Apart from the versatility, there are several theoretical advantages of SPAD in children in comparison to other liver support systems.

In contrast to MARS and high-volume plasma exchange with treatment sessions of up to $8 \mathrm{~h}$, SPAD operates as a CVVHDF, as used normally in critically ill and hemodynamically unstable patients. Hereby, detoxification occurs continuously with a lower risk of disequilibrium through excessive and intermittent toxin removal.

Further, it is a potential advantage of SPAD that blood flow rates, duration of therapy, and albumin concentration can be individually tailored for each patient. Although we still ignore the optimal dose for the individual patient, this hypothesis is supported by a meta-analysis on albumin dialysis which showed a non-significant trend towards risk reduction in mortality only in studies with more dialysis sessions [25].

A clinically relevant advantage of SPAD is the low extracorporeal volume allowing the application even in small children as shown in our cohort with a median weight of $11 \mathrm{~kg}$. The disposal of albumin after single use is an apparent disadvantage of the technique. On the contrary, there is evidence for a decline of the albumin concentration during MARS therapy due to the binding of albumin to the adsorbent columns [28]. Furthermore, the costs for $24 \mathrm{~h}$ SPAD are markedly lower than for a MARS system. With our settings (median dialysate flow $26 \mathrm{~mL} /$ $\mathrm{kg}^{*} \mathrm{~h}$ and median albumin concentration $4 \%$ ), around $125 \mathrm{~mL}$ human albumin $20 \%$ are used per kilogram patient's weight in $24 \mathrm{~h}$. In addition, in comparison to plasma exchange and hemodialysis, there is less exposure to foreign donor blood products, and thereby a reduced risk for allergic reactions and HLA sensitization.

The number of deceased patients raises concern whether SPAD is limited in stabilizing and bridging pa-
60

Blood Purif 2020;49:55-62

DOI: $10.1159 / 000502938$
Holle/Gratopp/Balmer/Varnholt/ Henning/Bufler/Müller/Rosenfeld 
tients to transplantation. The only severe complication, however, directly associated with SPAD occurred in a child with a body weight of $4.2 \mathrm{~kg}$ who suffered from pulmonary hemorrhage. The child recovered without sequelae. We expected low blood flow rates in small children to lead to a higher risk of clotting. Interestingly, patients' weights had no influence on the duration of the dialysis sessions and clotting in our cohort.

We accepted the risk of clotting over the risk of bleeding by starting dialysis without anticoagulation in $6 / 19$ (32\%) patients. No anticoagulation was inferior to heparin and RCA regarding filter life, whereas no significant difference in filter life between RCA and heparin anticoagulation was observed. Of note, RCA could be safely administered in patients with severe liver dysfunction. However, our cohort size was too small to evaluate the issue of adequate anticoagulation conclusively in patients with liver failure.

Major limitations of our observations are the retrospective design and the lack of control group. Thus, it is impossible to draw robust conclusions on mortality. Indications for the treatment might vary between different patients. A prospective, protocol-based approach would allow better comparisons of patients. The results of this analysis outline potentially fruitful areas of future clinical research. It is presently unclear which metabolites and toxins play a crucial role in ALF. Different substances with a possible role in HE like manganese, pro-inflammatory cytokines as well as mercaptans, short-chain fatty acids, GABA, glutamate and others could be investigated prospectively $[29,30]$.

In conclusion, we report about a pediatric cohort with liver failure, treated with SPAD. Although a direct comparison is impossible, efficacy seemed similar to other reports about extracorporeal liver support in adult patients or in children treated with MARS. Regarding documented direct adverse effects of SPAD, the method appeared safe. However, further investigations are needed to evalu- ate the effect on meaningful clinical outcomes, especially survival and long-term neurologic sequelae, as well as appropriate and individual dosage.

\section{Acknowledgements}

The work was performed at the Department of Pediatric Pulmonology, Immunology and Intensive Care Medicine, CharitéUniversitätsmedizin Berlin. We thank all nurses and doctors who were involved in patient care.

\section{Statement of Ethics}

The study was performed in accordance with the Federal and State Laws and recommendations of the local data safety office. The Institutional Review Board of Charité-Universitätsmedizin Berlin waived the need for approval due to the retrospective nature of the study.

\section{Disclosure Statement}

All authors declare that they have no conflicts of interest to disclose.

\section{Funding Source}

There was no funding supporting the present retrospective analysis which is solely investigator initiated work.

\section{Author Contributions}

J.B.H. and L.R.: conception and design. J.B.H., L.R., S.H., A.G., and V.V.: collection of data. J.B.H., L.R., and S.B.: analysis. J.B.H., L.R., and D.M.: interpretation of data. J.B.H. and L.R.: drafting of the article. J.B.H., L.R., D.M., and P.B.: revision of article. J.B.H., L.R., D.M., S.H., A.G., V.V., and P.B.: providing intellectual content of critical importance to the work described. J.B.H., A.G., S.B., S.H., V.V., D.M., P.B., and L.R.: final approval of the version to be published.

\section{References}

1 Ng VL, Fecteau A, Shepherd R, Magee J, Bucuvalas J, Alonso E, et al. Studies of Pediatric Liver Transplantation Research G: Outcomes of 5-year survivors of pediatric liver transplantation: report on 461children from a north american multicenter registry. Pediatrics. 2008;122(6):e1128-35.

2 Ash SR. Extracorporeal blood detoxification by sorbents in treatment of hepatic encephalopathy. Adv Ren Replace Ther. 2002;9(1):3-18.
3 Huang KW, Chao A, Chou NK, Ko WJ. Hepatic encephalopathy and cerebral blood flow improved by liver dialysis treatment. Int J Artif Organs. 2003;26(2):149-51.

4 Mitzner S, Loock J, Peszynski P, Klammt S, Majcher-Peszynska J, Gramowski A, et al. Improvement in central nervous system functions during treatment of liver failure with albumin dialysis MARS-a review of clinical, biochemical, and electrophysiologi- cal data. Metab Brain Dis. 2002;17(4):46375.

5 Mitzner SR. Extracorporeal liver support-albumin dialysis with the Molecular Adsorbent Recirculating System (MARS). Ann Hepatol. 2011;10(suppl 1):S21-8.

6 Gupta S, Fenves AZ, Hootkins R. The Role of RRT in Hyperammonemic Patients. Clin J Am Soc Nephrol. 2016;11(10):18728. 
7 Ringe $\mathrm{H}$, Varnholt V, Zimmering M, Luck W, Gratopp A, Konig K, et al. Continuous venovenous single-pass albumin hemodiafiltration in children with acute liver failure. Pediatr Crit Care Med. 2011;12(3):257-64.

8 Ferenci P, Lockwood A, Mullen K, Tarter R, Weissenborn K, Blei AT. Hepatic encephalopathy-definition, nomenclature, diagnosis, and quantification: final report of the working party at the 11th World Congresses of Gastroenterology, Vienna, 1998. Hepatology. 2002; 35(3):716-21.

9 Kamath PS, Kim WR; Advanced Liver Disease Study Group. The model for end-stage liver disease (MELD). Hepatology. 2007; 45(3):797-805.

10 McDiarmid SV, Anand R, Lindblad AS; Principal Investigators and Institutions of the Studies of Pediatric Liver Transplantation (SPLIT) Research Group. Development of a pediatric end-stage liver disease score to predict poor outcome in children awaiting liver transplantation. Transplantation. 2002;74: 173-81.

11 Bozanich NK, Kwo PY. Renal insufficiency in the patient with chronic liver disease. Clin Liver Dis. 2015;19(1):45-56.

12 Kreuzer M, Ehrich JH, Pape L. Haemorrhagic complications in paediatric dialysis-dependent acute kidney injury: incidence and impact on outcome. Nephrol Dial Transplant. 2010;25(4):1140-6.

13 Steiner C, Mitzner S. Experiences with MARS liver support therapy in liver failure: analysis of 176 patients of the International MARS Registry. Liver. 2002;22(suppl 2):20-5.

14 Auth MK, Kim HS, Beste M, Bonzel KE, Baumann U, Ballauff A, et al. Removal of metabolites, cytokines and hepatic growth factors by extracorporeal liver support in children. J Pediatr Gastroenterol Nutr. 2005;40(1):54-9.
15 Schaefer B, Schaefer F, Engelmann G, Meyburg J, Heckert $\mathrm{KH}$, Zorn $\mathrm{M}$, et al. Comparison of Molecular Adsorbents Recirculating System (MARS) dialysis with combined plasma exchange and haemodialysis in children with acute liver failure. Nephrol Dial Transplant. 2011;26(11): 3633-9.

16 Schaefer B, Ujszaszi A, Schaefer S, Heckert $\mathrm{KH}$, Schaefer F, Schmitt CP. Safety and efficacy of tandem hemodialysis and plasma exchange in children. Clin J Am Soc Nephrol. 2014;9(9):1563-70.

17 Rustom N, Bost M, Cour-Andlauer F, Lachaux A, Brunet AS, Boillot O, et al. Effect of molecular adsorbents recirculating system treatment in children with acute liver failure caused by Wilson disease. J Pediatr Gastroenterol Nutr. 2014;58(2):160-4.

18 Bourgoin P, Merouani A, Phan V, Litalien C, Lallier M, Alvarez F, et al. Molecular Absorbent Recirculating System therapy (MARS(R)) in pediatric acute liver failure: a single center experience. Pediatr Nephrol. 2014;29(5):9018.

19 Quintero Bernabeu J, Ortega Lopez J, Juamperez Goni J, Julio Tatis E, MercadalHally M, Bilbao Aguirre I, et al. The role of molecular adsorbent recirculating system in pediatric acute liver failure. Liver Transpl. 2018;24(2):308-10.

20 Novelli G, Rossi M, Morabito V, Pugliese F, Ruberto F, Perrella SM, et al. Pediatric acute liver failure with molecular adsorbent recirculating system treatment. Transplant Proc. 2008;40(6):1921-4.

21 Collins KL, Roberts EA, Adeli K, Bohn D, Harvey EA. Single pass albumin dialysis (SPAD) in fulminant Wilsonian liver failure: a case report. Pediatr Nephrol. 2008;23(6): 1013-6.
22 Kihtir HS, Yildirim HM, Yesilbas O, Duramaz BB, Sevketoglu E. Single-pass albumin dialysis in a child aged six months with phenobarbital poisoning. Turk Pediatri Ars. 2016 Dec 1;51(4):228-230.

23 Novelli G, Rossi M, Pretagostini M, Pugliese F, Ruberto F, Novelli L, et al. One hundred sixteen cases of acute liver failure treated with MARS. Transplant Proc. 2005;37(6):2557-9.

24 Novelli G, Rossi M, Pretagostini R, Poli L, Novelli L, Berloco P, et al. MARS (Molecular Adsorbent Recirculating System): experience in 34 cases of acute liver failure. Liver. 2002; 22(s2 Suppl 2):43-7.

25 Tsipotis E, Shuja A, Jaber BL. Albumin Dialysis for Liver Failure: A Systematic Review. Adv Chronic Kidney Dis. 2015;22(5):382-90.

26 Larsen FS, Schmidt LE, Bernsmeier C, Rasmussen A, Isoniemi H, Patel VC, et al. Highvolume plasma exchange in patients with acute liver failure: an open randomised controlled trial. J Hepatol. 2016;64(1):69-78.

27 Bañares R, Nevens F, Larsen FS, Jalan R, Albillos A, Dollinger M, et al.; RELIEF study group. Extracorporeal albumin dialysis with the molecular adsorbent recirculating system in acute-on-chronic liver failure: the RELIEF trial. Hepatology. 2013;57(3):1153-62.

28 Gong D, Ji D, Ren B, Tao J, Xu B, Ronco C, et al. Significant decrease in dialysate albumin concentration during molecular adsorbent recirculating system (M.A.R.S.) therapy. Int J Artif Organs. 2008;31(4):333-9.

29 Butterworth RF. Pathogenesis of hepatic encephalopathy in cirrhosis: the concept of synergism revisited. Metab Brain Dis. 2016;31(6): 1211-5.

30 Riggio O, Ridola L, Pasquale C. Hepatic encephalopathy therapy: an overview. World J Gastrointest Pharmacol Ther. 2010;1(2):5463. 